\title{
Epidemiology of spinal cord paralysis in Fiji: 1985-1994
}

\author{
Jagdish C Maharaj \\ Chief Medical Officer, Rehabilitation Medicine, Medical Rehabilitation Unit, Tamavua Hospital, Private Mail Bag, \\ Suva, Fiji Islands
}

\begin{abstract}
This descriptive analytical ten year (1985-1994) retrospective study assessed the pattern of spinal cord paralysis (SCP) in the Fiji Islands utilising medical rehabilitation hospital data. Fiji Islands is an archipelago of 300 islands in the south western Pacific with a multi-ethnic population of over three quarters of a million. Rehabilitation of all SCP is provided at the Medical Rehabilitation Unit (MRU). Data was collected from medical records of new SCP $(n=140)$ admitted to MRU and analysed with Epi Info 5 assessing associations between cause and other variables. The incidence of new SCP admitted to the MRU was $18.7 / \mathrm{million} /$ year. There were $75(53.6 \%)$ traumatic and $65(46.4 \%)$ non-traumatic SCP. The incidence varied according to gender and ethnicity with Fijian male being at the highest (41.85) risk. Amongst traumatic SCP, $38.7 \%$ were due to falls, $25.3 \%$ motor vehicle accidents, $20 \%$ sports, $8 \%$ shallow water dive and $4 \%$ each deep sea diving and others, whereas among non-traumatic SCP, $52.3 \%$ were due to unknown causes, $32.3 \%$ infections, $9.2 \%$ neoplasms and $6.2 \%$ others. The male/female ratio was $4: 1$. The $16-30$ year age group accounted for $35 \%$ of SCP. $31 \%$ had tetraplegia and $52.1 \%$ had complete lesions. The subset of the sample who experienced traumatic SCP were more likely to be employed, aged between 16-30 years at the time of paralysis and to have complete tetraplegia. Those who experienced incomplete paraplegia were more likely to be unemployed, aged 46-60 years and educated to primary level at the time of paralysis. There was a high proportion of complete spinal lesion when compared with other studies. The incidence of secondary complications such as pressure sores and UTI was also found to be high when compared with other studies. The results support the view that young Fijian males are most prone to sustaining traumatic spinal cord paralysis, and that there is a high incidence of secondary preventable complications. The need for preventative measures and adequate rehabilitation are emphasised.
\end{abstract}

Keywords: spinal cord injury; Fiji; epidemiology; aetiology; incidence; non-traumatic paraplegia

\section{Introduction}

Spinal cord paralysis (SCP) results in profound and long-term disability or death. It is one of the most catastrophic lesions suffered by modern man, often leading to permanent paralysis. Multiple medical, social and vocational complications affect the victims who are usually young and in their most productive stages of life. This causes burden and suffering not only to the victim but to their families, to the health care system and to the community. The human and financial costs and implications of SCP are enormous. In the words of Sir Ludwig Guthmann 'of the many forms of disability which can beset mankind, a severe injury or disease of the spinal cord undoubtedly constitutes one of the most devastating calamities in human life'. ${ }^{1}$ Sir George Bedbrook reiterated that 'although spinal medicine has made much advance, persons afflicted with paralysis (traumatic or nontraumatic) of spinal cord origin constitute a major area of disability in every country and were neglected by most communities until the mid forties (and later) of this century, and this disability is today still the greatest handicap man can sustain'. ${ }^{2}$ The 'lifetime' costs for paraplegia approaches $\$ A 1,000,000$ and for tetraplegia $\$ A 2,000,000$ and enormous challenge and tragedy is associated with permanent paralysis. ${ }^{3}$ Many developing countries either have no or very little appropriate health care services for persons with SCP. Several studies from developed countries report motor vehicle accident as the leading cause of traumatic SCP. ${ }^{4}$ I reviewed hospital records of all SCP admitted to the Medical Rehabilitation Unit (MRU) in Fiji for the 10 year period between 1 January 1985 and 31 December 1994.

Fiji is an archipelago of about 300 islands in the south western Pacific straddling the international dateline and lying between 15 degrees and 22 degrees south of the equator. Fiji has a multi-ethnic community made up of native Fijians, Indians, and 
other ethnic groups. The total estimated population in 1991 was 783,232 made up of $46.7 \%$ ethnic Fijians, $48.3 \%$ ethnic Indians and $5 \%$ other races. ${ }^{5}$ Fiji's evolving economy is changing the agricultural-based to a buoyant tourism-based economy, accounting for approximately $14 \%$ of the Gross Domestic Product (GDP) in 1993, sugar trade was (12\% GDP), thriving industries and abundant natural resources. Tourism is the major foreign exchange earner but revenues from sugar, timber, gold, fish products and manufactured goods are steadily increasing and domestic entrepreneurship is thriving. ${ }^{6}$

The main provider of health care in Fiji is the Ministry of Health with a well developed geoadministrative infra-structure to deliver its services offering preventive, promotive, curative and rehabilitative health services. There are three large medical divisions with a base hospital in each one of them supported by smaller sub-divisional hospitals and health centres. The Ministry established its first 45 bed national MRU in 1984 at the Tamavua Hospital in the capital city, Suva where medical rehabilitation of all persons with SCP from whole of the nation is provided. It is a government funded facility providing free medical care to the public of Fiji. All traumatic and most non-traumatic SCP are first admitted to one of the three base hospitals then, after the acute phase, are transferred to the MRU for further management and continuing care. The MRU caters for: all persons with SCP; selected persons with stroke; many cases of multiple fractures, debilitating and neurodegenerative diseases, head trauma and amputees for limb fitting and gait training. Prior to 1984 , medical rehabilitation was generally limited to non-specialised services provided mainly at the hospitals and by some nongovernmental organisations (NGO). The operational philosophy of the Unit is based on the multi and interdisciplinary concept and emphasis is placed on development of community based rehabilitation programs. The MRU is staffed by three full-time doctors, a team of nursing staff and nurse-aides, physiotherapists, volunteer occupational therapists and social workers, part-time speech pathologist with regular cooperation from other volunteers and nongovernmental organisations.

\section{Method}

The purpose of this descriptive cross-sectional analytical study investigating for association between SCP and demographic, epidemiological and medical characteristics, including such factors as age, gender, ethnicity, occupation, education, aetiology, level and completeness of the lesion and medical complications, was to look at the pattern of SCP in Fiji in order to understand the magnitude of the problem through the analysis of 10 years, from 1 January 1985 to 31 December 1994 inclusive, of hospital records of people with SCP admitted to the MRU. All people with SCP admitted to the MRU at the Tamavua Hospital during the study period were included. Cases excluded from the study were people with SCP prior to 1 January 1985 but admitted to the MRU during the period of the study, congenital SCP (eg spina bifida) occurring prior to the period of the study, SCP that occurred during the study period but were not admitted to the MRU, including persons who died either at the other hospitals or pre-hospitals and were never transferred to the MRU and those who had rapid recovery not requiring specialised care and were discharged home from the acute hospitals. A case of SCP was defined according to the international classification adopted by the American Spinal Injury Association (ASIA). ${ }^{7}$

The data was collected from patients' hospital records kept at the MRU records office at the Tamavua Hospital in Suva, Fiji. This was done by myself by filling in the questionnaire for each case record, containing prospectively collected demographic, epidemiologic and medical variables. Epi Info 5 statistical software was used for analysis. ${ }^{8}$ Because there is variation in the rates of SCP due to traumatic and non-traumatic causes statistical analysis looked at patterns of SCP according to cause, differentiated into traumatic and non-traumatic for different age distribution, gender, ethnicity, educational and occupational groups, marital status, type, level and completeness of the lesion, length of stays in acute as well as rehabilitation, neurological/functional improvement, bladder management on admission and discharge, complications on admission, mortality and places of discharge. Strength of associations between variables was assessed to pin-point areas of major concerns. Significance tests were applied wherever appropriate. Categorical data was analyzed using Chi-square and $P$ values obtained.

\section{Results}

The number of people with SCP admitted to the MRU over the 10 year period between 1985 to 1994 ranged from 18 in 1985 to 54 in 1992, with an average of 34.4 per year, including the re-admissions. There were 140 individuals who met the inclusion criteria.

\section{Incidence}

The incidence of SCP ranged from a low of 11.2/ million/year in 1986 to a high of $29.4 /$ million/year in 1991. The average incidence for the 10 year period is estimated at $18.7 /$ million/year for both traumatic and non-traumatic causes combined. The incidence rates calculated separately were $5.6 /$ million/year each for 1986, 17.9 and 11.5 for 1991 , and averaged 10.0 and $8.7 /$ million/year over the 10 year period for traumatic and non-traumatic causes respectively. The rate of SCP varied according to gender and ethnicity. Fijian males had the highest rate of $41.85 /$ million/year followed by 35.93 for males of other races, 19.91 for Indian males, 12.34 for Fijian females, 11.18 for other females and 
3.47 for Indian females. This is illustrated in Figure 1 The data clearly shows a high incidence of 41.95/ million/year for Fijian males followed closely at 35.93 for other males, lowest being that of 3.47 among Indian females.

\section{Causes of spinal cord paralysis}

The causes of SCP in this sample were assessed as: traumatic, including motor vehicle accident, sports, shallow water dive, deep sea diving, falls and others; and non-traumatic, including spina bifida, tumour, infection, cause unknown and others. There were $53.6 \%$ (75) people with SCP due to traumatic causes and $46.4 \%$ (65) due to non-traumatic. The most frequent cause of traumatic SCP was falls $20.7 \%$ $(n=29)$, followed by motor vehicle accidents $13.6 \%$ $(n=19)$, sports $10.7 \% \quad(n=15)$, shallow water dive $4.3 \%(n=6)$, and deep sea diving and others $2.1 \%$ $(n=3)$ each. The most frequent cause of non-traumatic SCP was from unknown causes $24.3 \% \quad(n=34)$ followed by infectious causes $15.0 \%(n=21)$, tumour $4.3 \%(n=6)$ and others $2.9 \%(n=4)$. The comparative percentages of different causes of SCP admitted to the

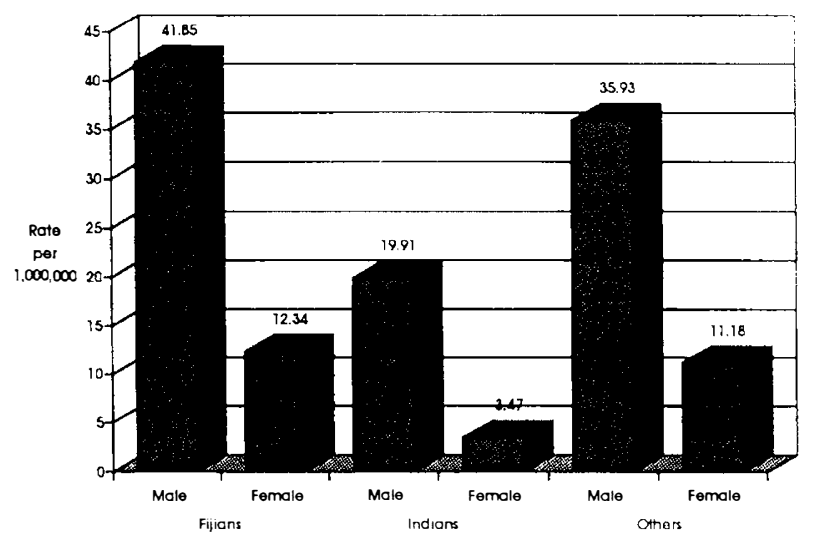

Figure 1 Incidence of SCP admitted to MRU 1985-1994 by sex and ethnicity

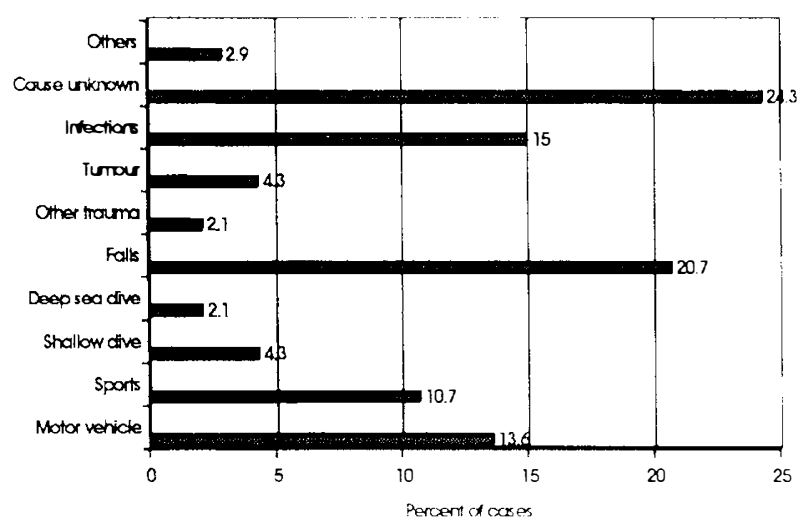

Figure 2 Percent of causes of spinal cord paralysis
MRU are presented in Figure 2. Since there was variation in the rates of SCP due to traumatic and non-traumatic causes the study factors were assessed for statistical significance against these.

\section{Gender and age}

There were $80 \%$ (112) male and $20 \%$ (28) female with SCP, the male/female ratio being $4: 1$. Sixty-five male $(87 \%)$ and 10 female $(13 \%)$ had traumatic and 47 male $(72 \%)$ and 18 female $(28 \%)$ non-traumatic SCP. The age at the time of paralysis ranged from 6 to 76 years with a mean of 38.3 years and standard deviation (SD) of 17.3. The average age for males was 38.7 years and that of females was 37.0 years. The rate of SCP in different age groups was as follows: $0-15$ years: $5.7 \%$ $(n=8) ; 16-30$ years: $35 \%(n=49) ; 31-45$ years: $24.3 \%$ $(n=34)$; $46-60$ years: $22.1 \%(n=31)$ and $61+$ years: $12.9 \%(n=18)$. Figure 3 illustrates the age distribution of people with SCP by age group and gender.

The age group 16-30 years accounted for highest numbers of people with SCP for both genders and the age group 16-45 years accounted for $59.3 \%$ of all people with SCP admitted to the MRU 1985-1994. The $0-15$ year age group had $62.5 \%(5 / 8)$ affected by infectious causes. The $16-30$ year age group has a bimodal pattern with sports and falls accounting for $24.5 \%(12 / 49)$ of people with SCP each. In the age group $31-45$ years $23.5 \%(8 / 34)$ were due to motor vehicle accidents, $17.6 \%(6 / 34)$ due to falls followed closely at $14.3 \%(5 / 34)$ each due to infectious and unknown causes. The unknown causes accounted for the highest number of $38.7 \%(12 / 31)$ and $35.5 \%(11 /$ $31)$ in the $46-60$ and $61+$ year age groups respectively. The distribution according to age group and cause of paralysis is presented in Table 1. The 16 - 30 year age group accounted for $50.7 \%(38 / 75)$ of traumatic causes and $46-60$ year age group for $35.4 \%$ $(23 / 65)$ of non-traumatic causes of SCP. There was a highly significant association $\left(\chi^{2}=27.44, \quad P<0.001\right)$ between age group and cause with less than 30 years more likely to sustain traumatic (falls, sports) and over 46 years non-traumatic of unknown cause SCP.

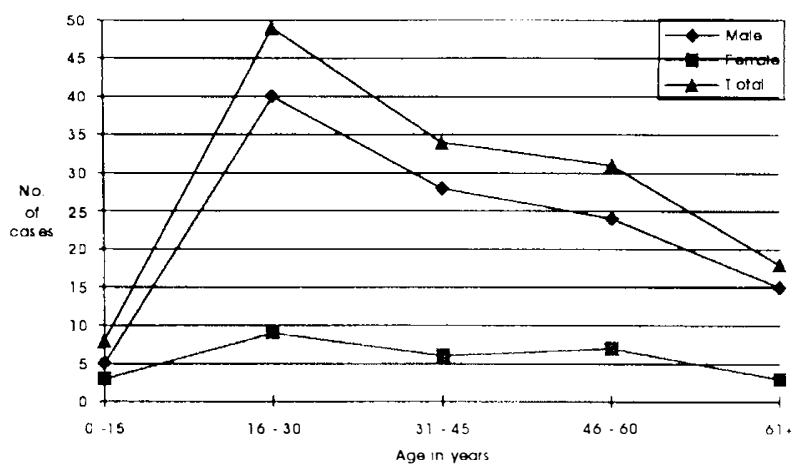

Figure 3 Distribution of spinal cord paralysis by age group and gender 


\section{Ethnicity}

Fiji being a multi-ethnic nation, an ethnic breakdown of people with SCP admitted to the MRU is presented. The study sample comprised of $64.3 \%$ (70 male, 20 female) Fijians, $29.3 \%$ (35 male, six female) Indians, and $6.4 \%$ (seven male, two female) others. There were equal numbers of 45 Fijians with SCP due to traumatic and non-traumatic causes each. There were 26 Indians with SCP due to traumatic and 15 non-traumatic causes. For the other races there were four due to traumatic and five non-traumatic causes.

\section{Education and occupation}

The majority $[61.4 \%(86)]$ of the people with SCP had only primary school level education, $28.6 \%$ (40) secondary and only $10.0 \%$ (14) had tertiary level of education at the time of paralysis. Traumatic causes accounted for 35, 31 and nine people with SCP, and non-traumatic causes for 51, nine and five people with SCP from primary, secondary, and tertiary level of education respectively. There was a significant association $\left(\chi^{2}=15.58, P<0.001\right)$ between level of education and whether the SCP was due to traumatic or nontraumatic cause.

The occupational status of the sample, at the time of paralysis, showed that $17.1 \%$ (24) were students, $30.0 \%$ (42) were employed, $42.1 \%$ (59) were unemployed and $10.7 \%$ (15) had retired. Traumatic causes accounted for $12,32,28$ and three people with SCP and non-traumatic causes for $12,10,31$ and 12 people with SCP with occupational status of students, employed, unemployed and retired respectively. There was also a significant $\left(\chi^{2}=16.45, P<0.001\right)$ association between occupational status and whether the SCP was due to traumatic or non-traumatic cause.

\section{Marital status}

Fifty-nine point three percent (83) were married, 37.1\% (52) (includes children and adolescents) were never married and $3.6 \%$ (5) were widowed at the time of paralysis. There were 31 people with traumatic SCP who were never married, 43 married and one widowed

Table 1 Spinal cord paralysis by age group and cause

\begin{tabular}{|c|c|c|c|c|c|c|}
\hline Cause & $0-15$ & $\begin{array}{l}\text { Age gi } \\
16-30\end{array}$ & $\begin{array}{l}\text { oup in } \\
31-45\end{array}$ & $\begin{array}{l}\text { years } \\
46-60\end{array}$ & $60+$ & Total \\
\hline Motor vehicle & 0 & 9 & 8 & 1 & 1 & 19 \\
\hline Sports & 0 & 12 & 3 & 0 & 0 & 15 \\
\hline Shallow water dive & 1 & 3 & 2 & 0 & 0 & 6 \\
\hline Deep sea diving & 0 & 1 & 1 & 1 & 0 & 3 \\
\hline Falls & 1 & 12 & 6 & 6 & 4 & 29 \\
\hline Others (trauma) & 0 & 1 & 1 & 0 & 1 & 3 \\
\hline Tumour & 0 & 0 & 3 & 2 & 1 & 6 \\
\hline Infection & 5 & 6 & 5 & 5 & 0 & 21 \\
\hline Cause unknown & 1 & 5 & 5 & 12 & 11 & 34 \\
\hline Others & 0 & 0 & 0 & 4 & 0 & 4 \\
\hline Total & 8 & 49 & 34 & 31 & 18 & 140 \\
\hline
\end{tabular}

and among people with non-traumatic SCP 21 were never married, 40 married and four widowed.

\section{Neurological level of lesion}

There were $30.7 \%$ (43) SCP above the first thoracic (T1) level resulting in tetraplegia/paresis and $69.3 \%$ (97) below T1 resulting in paraplegia/paresis. During the 10 year study period, there was only one person with high (C1-4) level tetraplegia, 30.0\% (42) with low level (C5-T1) tetraplegia/paresis and amongst the paraplegic/paretic $10.0 \%$ (14) were with high thoracic (T2-6) level, 37.9\% (53) lower thoracic (T7-12) level and $21.4 \%$ (30) lumbosacral (L-S) level SCP admitted for medical rehabilitation. This is illustrated in Figure 4.

There were 40 tetraplegia/paresis and 35 paraplegia/ paresis due to traumatic and three tetraplegia/paresis, and 62 paraplegia/paresis due to non-traumatic causes. There was a highly significant $\left(\chi^{2}=38.83, P<0.001\right.$, $\mathrm{OR}=23.62, \mathrm{CI}=6.28-104.85)$ association between the neurological level of the lesion and cause with trauma more likely to result in tetraplegia/paresis, whereas non-trauma in paraplegia/paresis.

\section{Completeness of the lesion}

Fifty two point one percent (73) were complete and $47.9 \%$ (67) were incomplete SCP. Of these, there were 46 complete and 29 incomplete SCP due to traumatic causes, and 27 complete and 38 incomplete SCP due to non-traumatic causes. There was a significant $\left(\chi^{2}=5.47, P<0.01, \quad \mathrm{OR}=2.23, \mathrm{CI}=1.07-4.67\right)$ association between completeness of the lesion and whether the lesion was caused by traumatic or non-traumatic agent. There was a 2.23 times higher risk of having a complete lesion due to traumatic causes. There were 23 tetraplegic and 20 tetraparetic and 50 paraplegic and 47 paraparetic people with SCP admitted to the MRU.

\section{Urinary bladder management}

On admission $18.6 \%$ (26) of people with SCP had normal bladder function, $77.1 \%$ (108) had an

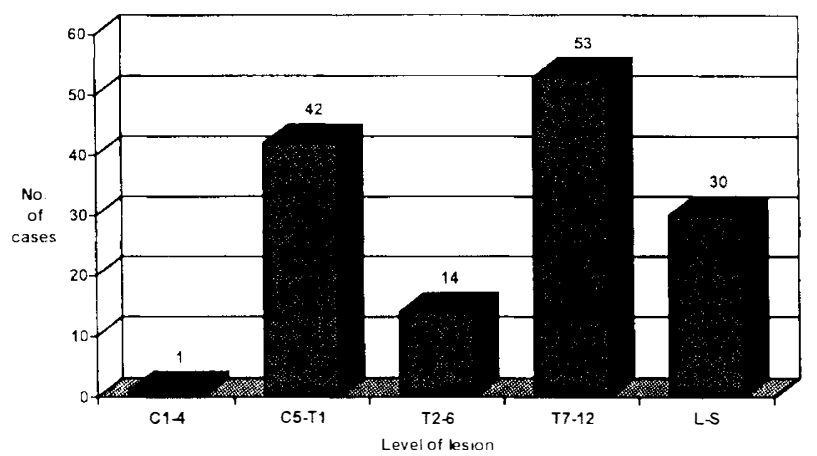

Figure 4 Distribution according to level of paralysis 
indwelling urinary catheter, $3.6 \%$ (5) were using suprapubic Crede pressure method for emptying their bladder and only $0.7 \%$ (1) had a suprapubic catheter. At discharge from the MRU 36.0\% (45) had attained normal bladder function, 33.6\% (42) had an indwelling urinary catheter, $12.0 \%$ (15) were using condom catheter, $12.8 \%(16)$ were doing their intermittent self catheterisation, $4.8 \%$ (6) were using Crede method and $0.8 \%$ (1) had suprapubic catheter. Only 125 people with SCP were included in the bladder management at 'discharge' from the Unit compared to 140 on admissions as 15 people had died prior to discharge. The urinary bladder management methods at discharge from the MRU as compared to that on admission had a large decrease in the number of indwelling catheter use and an increase in normal bladder, condom catheter and intermittent self catheter use as illustrated in Figure 5. The data shows that the percent of urinary bladder with normal function doubled and the use of indwelling urethral catheters halved after medical rehabilitation. Also the use of condom catheter, Crede and intermittent self catheterisation increased.

\section{Medical complications}

Twenty-nine point three percent (31) of people with SCP were admitted to the MRU free from any complications and the rest of the $70.7 \%$ (99) were admitted with various complications. These included $15 \%(n=21)$ pressure sores alone, $5.7 \%(n=8)$ urinary tract infection (UTI) alone, $2.9 \%(n=4)$ spasticity alone and $47.1 \%(n=66)$ with multiple combinations of complications like pressure sores, UTI, urethral stricture or fistula, skin and chest infections, osteomyelitis and spasticity. This is illustrated in Figure 6. There were 17 traumatic and 24 non-traumatic SCP free of any complications, and 58 traumatic and 41 non-traumatic had either one or multiple complications. Among the complete lesions 67 had multiple complication and only six were free from any complication; and among incomplete lesions 32 had multiple complications and 35 were free of any

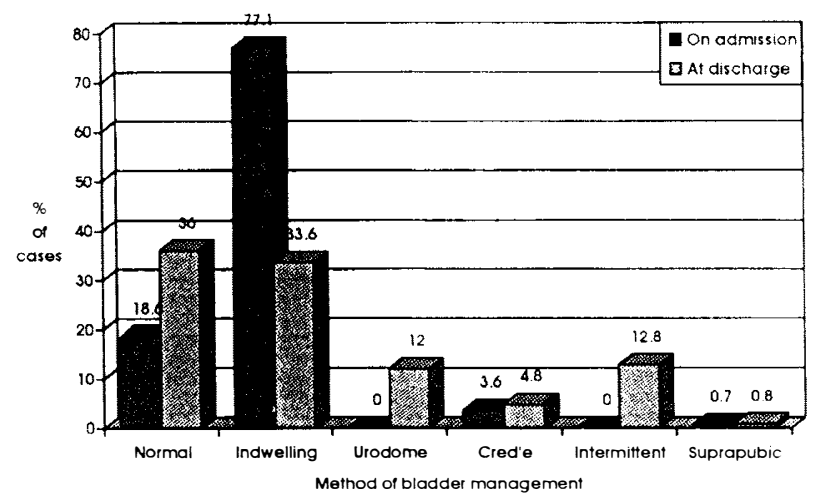

Figure 5 Urinary bladder management on admission and at discharge complication. There was a highly significant association $\left(\chi^{2}=32.69, \quad P<0.001, \quad \mathrm{OR}=12.21, \quad \mathrm{CI}=4.33-\right.$ 36.24) between occurrence of complication and whether the lesion was complete or incomplete. Complete lesions were 12 times more likely to have come to MRU with medical complications.

\section{Neurological/functional improvement}

Thirty-one point four percent $(n=44)$ of people with SCP had neurological/functional improvement as assessed at discharge compared with the status at admission and the rest of $68.6 \%(n=96)$ remained the same. Of the 73 complete SCP, five improved and 68 did not, whereas of the 67 incomplete SCP 39 improved and 28 did not. As expected, there was a highly significant association $\left(\chi^{2}=42.76, \quad P<0.001\right.$, $\mathrm{OR}=18.94, \quad \mathrm{CI}=6.27-61.40) \quad$ between neurological $/$ functional improvement and completeness of the lesion. The incomplete lesions show almost 19 times more chance of improvement compared to the complete lesions.

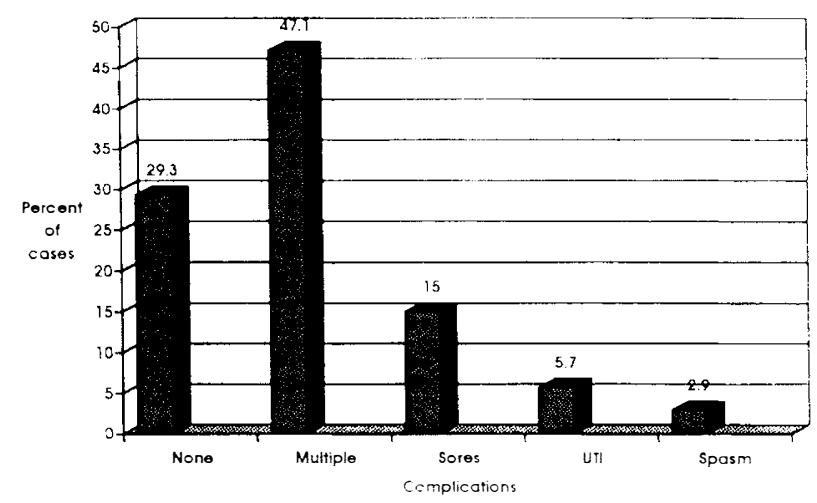

Figure 6 Medical complications present on admission to MRU

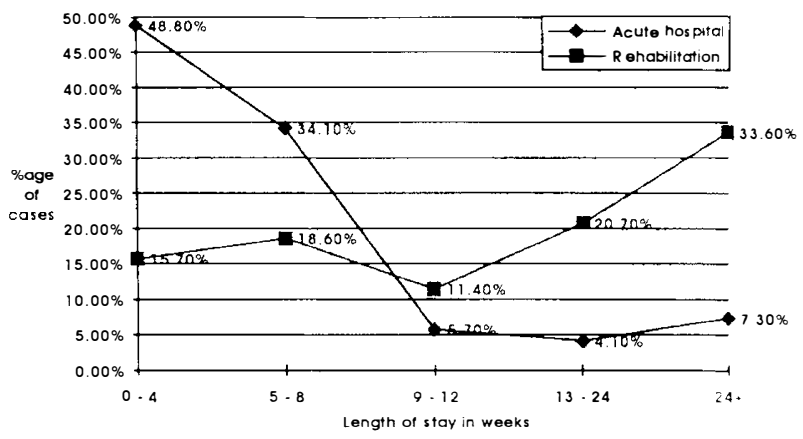

Figure 7 Distribution of length of stays in acute and rehabilitation hospitals 
Length of stay

Of the 123 people with SCP that were admitted to the MRU via the acute hospitals $48.8 \%(n=60)$ had $0-4$ weeks length of stay (LOS) in acute hospitals, 34.1\% $(n=42)$ had 5-8 weeks, 5.7\% $(n=7)$ had $9-12$ weeks, $4.1 \%(n=5)$ had $13-24$ weeks and $7.3 \%(n=9)$ had more than 24 weeks before being transferred to the MRU. In the MRU the LOS were $15.7 \%(n=22) 0-4$ weeks, $18.6 \%(n=26) 5-8$ weeks, $11.4 \%(n=16) 9-12$ weeks, $20.7 \%(n=29) 13-24$ weeks and $33.6 \%(n=47)$ more than 24 weeks. The acute hospital LOS compared to that of the MRU are mirror images of each other. A high proportion $(48.8 \%)$ of people with SCP spent less than 4 weeks in acute hospitals with only $7.3 \%$ spending more than 24 weeks, whereas $15.7 \%$ spent less than 4 weeks in MRU with $33.6 \%$ spending more than 24 weeks. This is illustrated in Figure 7.

\section{Mortality}

During the study period there were 15 deaths in the MRU (mortality rate of $10.7 \%$ ) comprising of 14 complete and only one incomplete spinal cord lesion. These were seven traumatic and eight non-traumatic SCP, six being tetraplegic and nine paraplegic, 13 male and two female; and 12 Fijians and three Indians. Three were in 16-30 year age-group, three in 31-45 year age-group, four in 46-60 year age-group and five were over 61 years.

\section{Discharges}

Seventy-five percent (105) of people with SCP were successfully discharged into the community to their own homes, $10.7 \%$ (15) died in the MRU, 6.4\% (9) needed transfer to acute hospitals from where they did not return being either discharged home or died, $3.6 \%$ (5) had to be re-housed in the Housing and Relief Trust (HART) homes and $4.3 \%$ (6) remained in the MRU at 2 year follow-up. Fifty-eight traumatic and 47 non-traumatic SCP were discharged to their own homes in the community, whereas 17 traumatic and 18 non-traumatic SCP met the other 'discharge' status.

There was no statistically significant association (at $\alpha=0.5$ ) between gender, ethnicity, marital status, completeness of the lesion, medical complications, neurological/functional improvement or place of discharge and whether the SCP was due to traumatic or non-traumatic cause.

\section{Discussion}

The 140 people with new SCP admitted to the MRU during the 10 year study period 1985-1994 corresponds to an overall incidence of $18.7 /$ million/year. The incidence due to traumatic causes was 10.0 and non-traumatic causes $8.7 / \mathrm{million} / \mathrm{year}$. The incidence of SCP varies when comparing different reports. Reviewing the medical literature published from 1975 to 1990 Shingu et al reported the incidence of the newly hospitalised patients with traumatic SCP to range from 9.2 to 53.4 per million per annum. ${ }^{4}$ A study in Hualien county Taiwan, which excluded the patients who died before hospitalisation, the incidence was still as high as $56.1 /$ million/year. ${ }^{9}$ The incidence of 10.0 derived from this study lies at the lower end of the 9.2 to 53.4 range with incidence of only Denmark being less than that of Fiji. The incidence in Japan was similar to that of recent studies in Australia, the Federal Republic of Germany and the USA, which were reported to range from 27.6 to 36.0. An incidence of 8.7 for nontraumatic SCP was reported in a three year retrospective review of 150 cases of new SCP admitted to hospitals in Monroe county in New York of 23.8/ million/year due to cancer, 15.2 due to spondylotic myelopathy, 12.9 due to benign tumour, intraspinal abscess, vascular injury, transverse myelitis and epidural haematoma. ${ }^{10}$ This study used rehabilitation facility data which is expected to yield a lower incidence as people with SCP who were referred and admitted to the facility only are taken into account. Those who died pre-hospital or at the other hospitals before being admitted to the MRU are not included. Thus the true incidence of SCP in Fiji could be expected to be higher.

Traumatic causes accounted for $53.6 \%$ and nontraumatic for $46.4 \%$ of SCP. This study sample from Monroe county showed $27.3 \%$ traumatic and $72.7 \%$ non-traumatic causes. ${ }^{10}$ This could be explained by the higher mean age of $54.4 \pm 21.6$ years for their sample. On the other hand, a study of 616 cases with majority in the age group 20-29 years from South Africa showed that $89 \%$ were due to traumatic and only $11 \%$ non-traumatic causes, with acts of violence accounting for $55 \%$ of the cases. ${ }^{11}$ The result shows that falls $(38.7 \%)$ account for the highest proportion of traumatic SCP followed by motor vehicle accidents $(25.3 \%)$, sports $(20 \%)$, shallow water dive $(8 \%)$, deep sea diving $(4 \%)$ and $(4 \%)$ other causes. Falls from a height and falls on the ground level are not distinguished. Majority of the falls were from breadfruit, mango and coconut trees and buildings and houses. The status of victims being passenger, driver or pedestrian in motor vehicle accidents is also not distinguished. The 15 cases resulting from sports were all males due to rugby football except one which was due to soccer. The shallow water dives were from rivers, sea and swimming pools. All the paralysis due to deep sea diving comprised of spinal Decompression Sickness from diving for beche-der-mer in deep waters with the 'Hooker' diving apparatus.

Shingu et al also compared the causes of spinal cord injuries as reported in the literature for the 1975-1990 period for Australia, Florida and Taipei with that of Japan. ${ }^{4}$ They showed that road traffic accident consistently comprised the main cause of spinal cord injury with fall as the next greatest cause. Studies from Brazil $^{12}$ (traffic accident $41.7 \%$, falls $14.8 \%$ ), Taiwan ${ }^{9}$ (traffic accident $61.6 \%$, falls $23.3 \%$ ) and Utah, USA ${ }^{13}$ (motor vehicle crashes $49.3 \%$, falls $21.1 \%$ ) show 
motor vehicle accidents as the leading cause of traumatic SCP followed by falls. On the other hand, studies from India ${ }^{14}$ (falls $55.2 \%$, road traffic accidents $12.8 \%$ ), Nigeria ${ }^{15}$ (falls $48 \%$, road traffic accidents $36 \%$ ) and Romania ${ }^{16}$ (falls $59 \%$, road traffic accidents $13 \%$ ) show falls as the leading cause of SCP. Okonkwo has suggested that falls from trees in Nigeria may be related to seasonal weakening of the tensile strength of jigs woven from palm fronds and used for climbing palm trees. ${ }^{15}$ Fiji has largely an agricultural based economy and an abundance of seasonal fruiting which probably explains for the high incidence of SCP due to falls $(38.7 \%)$ from trees; and a well developing road network which contributes to motor vehicle accidents $(25.3 \%)$ as another cause.

The results also show that unknown cause $(52.3 \%)$ accounts for the highest proportion of SCP due to non-trauma. This is followed by infectious $(32.3 \%)$, tumours $(9.2 \%)$ and $(6.2 \%)$ other non-traumatic causes. Most of the SCP from the unknown causes were investigated at the acute hospitals but no cause was established. Some of these would have constituted cases of Transverse Myelitis. All the infectious cases were established as such. Most of these were epidural abscesses surgically decompressed. Some were diagnosed as Mycobacterium infection through radiological evidence and concomitant pulmonary disease. The cases due to tumour included both primary as well as secondary metastatic tumours.

Rudge et al studied 44 consecutive patients of AfroCaribbean origin in the United Kingdom diagnosed as myelopathy of unknown cause and of the 30 patients with progressive paraparesis $27(90 \%)$ had serum antibodies to human T-cell lymphotrophic virus type 1 (HTLV-1) and were classified as having tropical spastic paraparesis (TSP). ${ }^{17}$ Another study found 18 $(90 \%)$ out of 20 HTLV-1 seronegative neurological patients with symptomatology suggesting HTLV-1 associated myelopathy or tropical spastic paraparesis (HAM/TSP) tested positive for oligonucleotide pairs when polymerase chain reaction (PCR) was used. ${ }^{18}$ Gessain and Gout critically analysed voluminous literature from studies after 1985, when TSP/HAM was first recognised, included information from 250 publications and concluded that TSP/HAM is a common neurological disease in many parts of the world with a prevalence ranging from 5.1 to 128 per 100,000 inhabitants and that up to $20 \%$ of patients develop TSP/HAM after transfusion of HTLV-1 contaminated blood. ${ }^{19}$ The prevalence of HTLV-1 is unknown in the Melanesians (Fijians) but was found to be $2.2 \%$ in the Solomon Islands (Micronesians). ${ }^{20}$ Another study in several remote indigenous populations of the Solomon Islands and Vanuatu (Banks and Torres islands) and in some coastal and inland groups in Papua New Guinea and Irian Jaya suggested possible different strain or a variant of HTLV-1 or a HTLV-1-related retrovirus present in Melanesia. ${ }^{21}$ This study sample had no contact with Japanese or Africans and little contact with white persons before blood samples were collected and demonstrated extremely high IgG antibody titres to HTLV-1 being $1: 5120$ in serum and $1: 160$ in cerebrospinal fluid.

Although there is no previous study of prevalence or incidence of spinal cord paralysis from Fiji, a series of four patients with conversion disorder presenting as spinal cord paralysis who met the diagnostic criteria of the Diagnostic and Statistical Manual III and came from a single hospital (CWM Hospital, Suva, Fiji) in a short span of time within the same year was reported. $^{22}$ A 12 month prospective study at the Prince Henry Hospital in Sydney found a surprisingly high proportion of $9.8 \%$ of their acute spinal cord injuries diagnosed as conversion disorder. ${ }^{23}$ In this series the mean age was $23 \pm 8$ years, the male/female ratio $8: 1$ and more than $50 \%$ had a previous history of conversion disorder. When investigating the "cause unknown' SCP the possibility of conversion disorder and HTLV-1 should also be considered in Fiji population.

The result shows that the study sample had $80 \%$ (112) male and $20 \%$ (28) female, the male to female ratio being $4: 1$. This is similar to that reported in the literature from Taiwan ${ }^{9}$ and South Africa ${ }^{11}(4: 1)$, Brazil $^{12}(3.9: 1)$ and Turkey ${ }^{24}(11: 3)$. The age ranged from 6 to 76 years with a mean of $38.3 \pm 17.3$ years. The mean age for males was 38.7 and that of females was 37.0 years. Other studies reported age range from 6 to 56 years with a mean of $30.3 \pm 1.1$ years and $75.9 \%$ cases in the 11 to 40 year age group from Brazil, ${ }^{12}$ whereas in Japan $^{4}$ the age ranged 6 to 96 years with a mean of $48.3 \pm 19.5$ years with males $48.3 \pm 19.6$ and females $49.2 \pm 19.3$ years. The life expectancy of the country should influence the overall age range but in Fiji the age group 16-30 years comprised the largest $(35.0 \%)$ with $16-45$ year age group accounting for $59.3 \%$ of cases. Thus the age group 16 to 45 years seems to be at the highest risk. There was a significant association $(P<0.001)$ between age and causes of SCP with the younger age group more likely to sustain traumatic and the older age group non-traumatic SCP.

Fijians have the highest incidence followed by other ethnic groups and Indians. Fiji has a unique ethnic mix and only a couple of studies found in the literature reported the ethnic difference in the incidence. Dixon et al from New Zealand reported that although spinal cord injury occurs most often in young Caucasians as a result of motor vehicle accidents, ethnicity adjusted rates show high rates for Maori males and Price et al in their study in Oklahoma, USA report that SCP among blacks (as compared to the whites) as a result of violence, was 'remarkable'. ${ }^{25,26}$ The authors suggested that more complete reporting of SCP among blacks than among whites could account for some discrepancy as violence related SCP is more completely reported and that socioeconomic status may have a role. There is no study from a similar ethnic mix to Fiji to compare with these findings. Nonetheless, in Fiji, probably the 
nature of activities of different ethnic groups could explain for the differences in incidence rates to some extent. Rugby football is almost exclusively played by Fijians. When the 14 people with SCP due to rugby, who were admitted to the MRU, are excluded the incidence rate for Fijians decreases from 41.85 to $33.48 /$ million/year which is less than the rate of 35.93 for the other races. Thus just one sport contributes significantly to the incidence of SCP among Fijians as compared to the other ethnic groups in Fiji. This sample does not include the fatal cases who were not admitted to the MRU, so the actual incidence will be even higher.

Data not adjusted for the proportion of persons with different levels of education in Fiji show that $61.4 \%$ had primary education at the time of paralysis. In Brazil ${ }^{12}$ majority of SCP had 'lower education level', whereas DeVivo et $a l^{27}$ studying the trend in spinal cord injury in USA showed that the percentage of high school graduates had increased significantly from 1973 to 1986 . They suggested that this trend could be due to increase in average age at injury. The results show that the majority $(42.1 \%)$ were unemployed at the time of paralysis followed by $30.0 \%$ employed, $17.1 \%$ students and $10.7 \%$ retired. Report from Romania ${ }^{16}$ show that $40 \%$ of the patients were manual workers, $12 \%$ intellectuals and $18 \%$ retired; and from Turkey ${ }^{24}$ the highest incidence was among housewives, followed by agricultural workers and private industry workers with the agricultural workers' wives group most at risk. Again these proportion may be explained by national proportions of the variable. The significant association $(P<0.001)$ of occupational status and cause could be a reflection of age distribution related to occupational status.

The majority $(59.3 \%)$ were married and $37.1 \%$ including children and adolescents were not married at the time of paralysis. A follow up study of spinal cord injuries in Iceland ${ }^{28}$ found that $35.6 \%$ were married before injury and in a large USA ${ }^{27}$ study for trend between 1973 and 1986 an average of $54.3 \%$ were single at the time of the paralysis. The USA study sample had a lower mean age of 29.5 years compared to that of this study of 38.3 years.

In this study there were $30.7 \%$ people who were tetraplegic and $69.3 \%$ paraplegic. These vary in different reports from only $8.1 \%$ with tetraplegia and $91.9 \%$ with paraplegia in Turkey, ${ }^{24} 28 \%$ and $72 \%$ in Nigeria, ${ }^{15} 38 \%$ and $62 \%$ in Spain, ${ }^{29} 51 \%$ and $49 \%$ in Denmark $^{30}$ to $71.7 \%$ and $28.3 \%$ in Naples. ${ }^{31}$ DeVivo et al reported a slightly increasing percentage of people with tetraplegia in a multi-centre study in the USA. ${ }^{27}$ The significant association between the level of the lesion and cause means that tetraplegia was more likely to result from trauma. The ratio of tetraplegia to paraplegia seems to be related to the cause of paralysis and will be affected by the survival rate of those with a high level of tetraplegia in different settings. There were $52.1 \%$ complete and $47.9 \%$ incomplete spinal cord lesions. Completeness of lesion was significantly associated with cause with trauma more likely to result in complete lesion. Again the ratio of complete to incomplete lesions vary in the literature. Chacko et $\mathrm{al}^{14}$ reported $72.8 \%$ and $27.2 \%$, Biering-Sorensen et $a l^{30} 48 \%$ and $52 \%$, Kiwerski ${ }^{32} 43.4 \%$ and $56.6 \%$ and Dixon et $a l^{25} 8 \%$ and $92 \%$ complete and incomplete spinal cord lesions respectively. Haffner et al reported equal proportions of complete and incomplete injuries in children of average age of 8.8 years. ${ }^{33}$ Completeness of the lesion seems to be related to the cause of paralysis with complete lesions more likely to result from trauma. Immediate first aid and pre-hospital care as well as the acute management must play a role in preserving or sparing of neurological function. This is also an area for further investigation as an incomplete lesion indicated a better survival and higher rehabilitation potential.

On admission to the MRU $18.6 \%$ of people with SCP had normal urinary bladder function and $77.1 \%$ had an indwelling urethral catheter compared with $36 \%$ and $33.6 \%$ respectively on discharge from the Unit. Intermittent self catheterisation was practised by $12.8 \%$ on discharge from the Unit. Garcia-Reneses et al reported $29 \%$ normal urinary bladder function, $20.8 \%$ urethral catheter use and $3.7 \%$ intermittent 'autocatheterism' in their series of retrospective study of 1010 cases for $1984-1985$ in Spain. ${ }^{29}$ In a selfreported mail questionnaire follow up study in Iceland of 45 people with SCP it was found that $55.6 \%$ did not have normal micturition, $20 \%$ were using Crede method, 52\% suprapubic tapping, $48 \%$ intermittent catheterisation and only $8 \%$ had indwelling urethral catheter. ${ }^{28}$ More than one method was employed by many subjects. A multi-centre study for trend from $1973-77$ to $1984-86$ in the USA found that the most common initial method of urinary bladder management was an indwelling urethral catheter, and this trend had somewhat decreased for 1984-86 in favour of increased use of intermittent catheterisation. ${ }^{27}$ This trend was true for both gender towards long term bladder management. In Fiji, although the percent of normal urinary bladder function had doubled and the use of indwelling urethral catheter halved, by discharge the use of intermittent self catheterisation and condom catheter are possibly limited by the availability of appropriate materials and adequate patient education. These are domains that are amenable to change and need further exploration.

The secondary medical complications can consume extensive resources, delay rehabilitation and cause further disability and handicap. The results show that on admission to the MRU $70.7 \%$ of the sample had some form of secondary medical complications. A total of $47.1 \%$ had combinations of multiple complications, $15 \%$ had pressure sores alone, $5.7 \%$ UTI, and $2.9 \%$ severe spasticity. Several authors report varying incidences of these complications. ${ }^{11,12,14,16,28,29}$ The most consistent of these seem to be pressure sores and urinary tract infection followed by respiratory infection, spasticity, pain and 
others. In this study there was a highly significant association between completeness of the lesion and the presence of complications on admission to the MRU. Complete lesions had 12 times the risk of developing complication. The most commonly reported complications of pressure sore and urinary tract infection are largely preventable and every effort should be made to prevent these from developing in acute, rehabilitative as well as after care phases. More investigation is required to assess the reasons for the failure of preventative measures.

Forty-four (31.4\%) people with SCP admitted to the Unit had neurological/functional improvement prior to discharge from the Unit. Biering-Sorenson et al reported that $41 \%$ of traumatic spinal cord injury patients had an improvement in their neurological status after their admission to rehabilitation hospital. ${ }^{30}$ This study demonstrated a significant association between improvement and completeness of the lesion with incomplete lesions 19 times more likely to show improvement. Ditunno et al in a study of 150 motor complete tetraplegic subjects recruited within 1 week of their injuries showed that the pattern of recovery in the key muscles of the 67 subjects with some motor power in the zone of partial preservation (incomplete lesion) was significantly $(P<0.001)$ greater than the 83 subjects with no motor power (complete lesion) at 3 to 6 months postinjury. ${ }^{34}$ Waters et al demonstrated that although the motor recovery rapidly declined in the first 6 months and then subsequently plateaued, $4 \%$ show 'late conversion' (more than 4 months after injury) from complete to incomplete spinal cord injury status. ${ }^{35,36}$ Hart and Williams reported recovery in $42 \%$ of their sample with stab wounds and nontraumatic lesions showing better rates of recovery than motor vehicle accidents and gunshot wounds. ${ }^{11}$ This is probably related to the extent of tissue damage caused by different agents. Although prevention is better than cure, when SCP does occur it is most important to have first aid, pre-hospital and acute facilities geared towards preserving whatever neurological sparing that may be present as an incomplete lesion has a much greater chance of neurological/functional recovery than a complete lesion.

Almost half of the cases $(n=60,48.8 \%)$ stayed in acute hospitals for less than 4 weeks with $7.3 \%(n=9)$ for more than 24 weeks, whereas in the MRU only $15.7 \%$ spent less than 4 weeks with $33.6 \%$ more than 24 weeks. It is not possible to directly compare this analysis with that from the literature as the reports of length of stay for people with SCP in the literature are from special centres for spinal cord injury where these patients receive full rehabilitation starting from the acute phase, unlike in Fiji where they are initially admitted to one of the three acute hospitals for stabilisation and acute care prior to post-acute rehabilitation at the MRU. Nonetheless, GarciaReneses et al $^{29}$ reported average time of hospitalisation in Spain as $4.6 \pm 0.2$ months $(18.4 \pm 0.8$ weeks) with a range of 1 to 14 months; Chacko et $a l^{14}$ reported average stay in hospital for tetraplegics as 62.7 days ( 9 weeks) and paraplegics as 42.3 days (6 weeks) and Hart and Williams ${ }^{11} 3-6$ months $(12-24$ weeks) in South Africa. In the USA there was a substantial decrease in mean total length of stay from 144.8 days (20.7 weeks) to 117.2 days (16.7 weeks) $(P>0.001) .{ }^{27}$ The length of stays at the MRU is largely determined by the level of the lesion, that is, tetraplegia or paraplegia, associated complications, staffing level, and the availability of appropriate equipment and family and community support.

The mortality rate at the MRU for the 10 year study period was $10.7 \%$. This is similar to that reported by Lan et al ${ }^{9}$ Soopramanien noted a mortality rate of $22 \%$ in $1985-1991$ period decrease to $10.1 \%$ in 1992 in Romania. ${ }^{16}$ Price et al found a $8 \%$ mortality rate in Oklahoma, USA. ${ }^{26}$ In this study and as reported in the literature majority of the deaths are due to renal, pulmonary and pressure sore complications. Elderly $(61+$ years $)$ Fijians with complete paralysis were at the highest risk. Seventyfive percent of people with SCP were discharged to their own homes into the community. There are no alternative forms of accommodation like nursing homes or special housing for people with SCP available in Fiji. For this reason 4\% remained in the MRU at 2 years from admission and another $4 \%$ were accommodated in Housing and Relief Trust (HART) homes. These homes are meant for poor and homeless people and not specifically for any disability group.

\section{Conclusion}

This study was conducted to explore the pattern of SCP in Fiji assessing the differences between traumatic and non-traumatic causes of paralysis. It is observed that, although non-traumatic 'unknown cause' accounts for the highest proportion $(24.3 \%)$ as a single category, overall, there are more cases of SCP due to trauma. Falls are the leading cause of traumatic SCP followed by motor vehicle accidents and sports, whereas infections are second only to unknown cause of non-traumatic SCP. There seems to be a need to screen patients presenting with unknown cause of SCP with features of HAM/TSP for HTLV-1 status. The incidence of some preventable secondary complications such as pressure sores and urinary tract infection are also high, further increasing disability, handicap and mortality. These and other complications of SCP must be prevented to reduce disability and handicap and improve survival and quality of life. This study has also demonstrated that a high proportion $(13 \%)$ of SCP were admitted to the MRU from their homes. Although it is not known as to what proportion of this $13 \%$ remained at their homes after the onset of paralysis or were discharged home from acute hospitals without the thought of further rehabilitation, medical rehabilitation of such a serious medical condition seems to remain an afterthought thus delaying the opportunity for timely interventions. 
The immense human suffering, disability and handicap, and the demands placed on the individual, the family, community, health care delivery system and the nation as a result of SCP calls for an urgent action towards prevention and appropriate management of the condition, if and when it does occur. There are numerous aspects of falls, motor vehicle accidents, sports and infections causing SCP amenable to prevention that should be addressed. Immediate first aid, pre-hospital and acute hospital care should aim to prevent any increase in the initial assault on the spinal cord by appropriate handling. This study has demonstrated a higher incidence of complete lesions indicating that there may be a missed chance of neurological preservation that could have provided a better rehabilitation potential and improved ultimate outcome. Early medical care should be meticulous and aimed at preventing progression of the lesion and development of preventable medical complications.

This study sample is from a medical rehabilitation facility thus reflecting the characteristics of only the people with SCP who were admitted for rehabilitation. It is likely that the picture would be different when pre-hospital and acute hospital samples are also taken into account. Nonetheless, this sample accurately reflects the population of people with SCP in Fiji who utilise medical rehabilitation health services.

Fiji has a well developed infrastructure, incorporating primary health care, for the delivery of health care services and a national MRU supporting both the hospital and primary health care systems. This study had demonstrated the need to give adequate emphasis to prevention of SCP and associated complications, and to provide appropriate medical care and rehabilitative management for persons with SCP. Appropriately trained staffed and adequately equipped care should be made available to persons with SCP to prevent and manage medical complications associated with the condition as well as providing comprehensive rehabilitation and continuing care in the community. The author is of the opinion that further development of health care services for people with disabilities, especially those with SCP, are urgently required and should be built upon the current services. The medical rehabilitation of persons with disabilities should be integrated with the primary health care delivery services as community based programs providing quality care with specialised support and coordination, at national level, from the MRU.

\section{Acknowledgements}

I wish to thank the staff of the Medical Rehabilitation Unit, particularly $\mathrm{Sr}$ Marseu Kaitu'u, for assistance towards this study and the Permanent Secretary for Health, Ministry of Health for permission to conduct and publish this study.

\section{References}

1 Thomas JP, Introduction. In: Young JS, Burns PE, Bowen AM, McCutchen R. (eds), Spinal Cord Injury Statistics-Experience of the Regional Spinal Cord Injury Systems. Good Samaritan Medical Center: Phoenix 1982. pp $1-7$.

2 Bedbrook GM. Keynote Address. In: Refshauge W (ed). Towards Prevention of Spinal Cord Injury - The Menzies Foundation Technical Report No. 1. Melbourne 1987. pp 9-12.

3 Yeo JD. Prevention of spinal cord injuries in an Australian study (New South Wales). Paraplegia 1993; 31: 759-763.

4 Shingu H, Ikata T, Katoh S, Akatsu T. Spinal cord injuries in Japan: a nationwide epidemiological survey in 1990. Paraplegia 1994; 32: 3-8.

5 Ministry of Health. Ministry of Health Annual Report 19911992, Fiji Paper No. 59 of 1993. Quality Print Ltd: Suva 1993.

6 Fiji Visitors Bureau. Fiji Visitors Bureau Publications. Fiji Visitors Burcau: Suva 1995.

7 Ditunno Jr JF, Young W, Donovan WH, Creasey G. The International Standards Booklet for Neurological and Functional Classification of Spinal Cord Injury. Paraplegia 1994; 32: $70-80$

8 Dean J, Dean A, Burton A, Dicker R. Epi Info Version 5.00 Center for Disease Control Office: Atlanta, USA 1990

9 Lan C et al. Traumatic spinal cord injury in rural region of Taiwan: an epidemiological study in Hualian county $1986-1990$. Paraplegia 1993; 31: $398-403$.

10 Murray PK, Kusior MF. Epidemiology of nontraumatic and traumatic spinal cord injury. Arch Phys Med Rehabil 1984; 65: 634.

11 Hart C, Williams E. Epidemiology of spinal cord injuries: a reflection of changes in South African society. Paraplegia 1994; 32: $709-714$.

12 Campos da Paz A et al. Traumatic injury of the spinal cord. Prevalence in Brazilian hospitals. Paraplegia 1992; 30, 636-640.

13 Thurman DJ et al. Surveillance of spinal cord injuries in Utah, USA. Paraplegia 1994; 32: 665-669.

14 Chacko V, Joseph B, Mohanty SP, Jacob T. Management of spinal cord injury in a general hospital in rural India. Paraplegia 1986; 24: $330-335$.

15 Okonkwo CA. Spinal cord injuries in Enugu, Nigeria preventable accidents. Paraplegia 1988; 21: $12-18$.

16 Soopramanien A. Epidemiology of spinal injuries in Romania. Paraplegia 1994; 32: $715-722$.

17 Rudge P, Ali A, Cruickshank JK. Multiple sclerosis, tropical spastic paraparesis and HTLV-1 infection in Afro-Caribbean patients in United Kingdom. Journal of Neurology, Neurosurgery \& Psychiatry 1991; 54: 689-694.

18 d'Auriol L et al. Diagnosis of HTLV-1 infected seronegative patients by polymerase chain reaction amplification in Martnique. Nouvelle Revue Francaise D'Hematologie 1990; 32: 113 116.

19 Gessain A, Gout O. Chronic myelopathy associated with human T-lymphotropic virus type I (HTLV-I) (Review). Annals of Internal Medicine 1992; 117: 933 -946.

20 Yanagihara $\mathrm{R}$ et al. Human T-lymphotropic virus type I infection in the Solomon Islands. American Journal of Tropical Medicine and Hygiene 1991; 44: $122-130$.

21 Ajdukiewicz A et al. HTLV-1 myeloneuropathy in the Solomon Islands (letter). New England Journal of Medicine 1989; 321: 615-616.

22 Kippax D, Maharaj JC. Conversion disorder presenting as spinal cord injury or disease. Fiji Medical Journal 1986; 14: 13-16.

23 Dickson H, Cole A, Engel S, Jones RF. Conversion reaction presenting as acute spinal cord injury. Medical J of Aust 1984; 141: $427-429$.

24 Dincer $\mathrm{F}$ et al. Traumatic spinal cord injury in Turkey. Paraplegia 1992; 30: $641-646$.

25 Dixon GS, Danesh JN, Caradoc-Davies TH. Epidemiology of spinal cord injury in New Zealand. Neuroepidemiology 1993; 12: $88-95$. 
26 Price $\mathrm{C}$ et al. Epidemiology of Traumatic spinal cord injury and acute hospitilisation and rehabilitation charges for spinal cord injuries in Oklahoma, 1988-1990. American Journal of Epidemiology 1994; 139: 37 - 47.

27 DeVivo MJ et al. Trends in spinal cord injury demographics and treatment outcomes between 1973 and 1986. Arch Phys Med Rehabil 1992; 73: 424-430.

28 Knutsdottir S. Spinal cord injury in Iceland 1973-1989. A follow up study. Paraplegia 1993; 31: 68-72.

29 Garcia-Reneses J, Herruzo-Cabrera R, Martinez-Moreno M. Epidemiological study of spinal cord injury in Spain 1984-1985. Paraplegia 1991; 28: $180-190$.

30 Biering-Sorensen E, Pedersen V, Clausen S. Epidemiology of spinal cord lesion in Denmark. Paraplegia 1990; 28: 105-118.
31 Palma V et al. Spinal cord injury: some epidemiological data. A review of 233 cases. Acta Neurologica 1992; 14: 29-38.

32 Kiwerski JE. The causes, sequelae and attempts at prevention of cervical spine injuries in Poland. Paraplegia 1993; 31: 527-533.

33 Haffner DL, Hoffer MM, Wiedbusch R. Etiology of children's spinal injuries at Rancho Los Amigos. Spine 1993; 18: 679-684.

34 Ditunno JF, Stover SL, Freed MM, Ahn JH. Motor recovery of the upper extremities in traumatic quadriplegia: A multicenter study. Arch Phys Med Rehabil 1992; 73: 413-416.

35 Waters RL, Yakura JS, Adkins RH, Sie I. Recovery following complete paraplegia. Arch Phys Med Rehabil 1992; 73: 784-789.

36 Waters RL, Adkins RH, Yakura JS, Sie I. Motor and sensory recovery following incomplete tetraplegia. Arch Phys Med Rehabil 1994; 75: $306-311$. 